Scientiæ studia, São Paulo, v. 11, n. 1, p. 221-42, 2013

\title{
2ิ \\ Considerações sobre o caminho original de Einstein rumo a uma teoria quântica da radiação (a propósito do artigo "Einstein y el efecto Compton”)
}

Michel PAтY

No artigo "Einstein y el efecto Compton", publicado neste número de Scientiæ studia, os autores estranham o fato de Einstein não ter declarado mais claramente o quanto esse efeito comprovava definitivamente o carácter corpuscular da radiação. A presente nota crítica pretende fornecer elementos adicionais de apreciação que permitam acompanhar o método de exploração do domínio dos quanta elaborado por Einstein, na ausência de uma teoria adequada, e praticado por ele de 1905 à 1925, evidenciando por esse meio propriedades inéditas e inusuais dos fenômenos quânticos; e também explicitar o alvo que ele queria almejar com sua investigação e entender melhor seu pensamento acerca dos quanta. Três argumentos principais são delineados. (1) O problema dos quanta de luz está estreitamente ligado com aquele das propriedades quânticas da matéria atômica em geral, do qual não pode ser separado. (2) Outro experimento, realizado pouco depois do experimento de Compton, trouxe um elemento adicional, necessário para que se possa atribuir sem nenhuma reticiência uma quantidade de movimento à radiação, isto é, o caráter individual da interação da qual a radiação toma parte. (3) Enfim, os trabalhos de Einstein sobre a estatística quântica, realizados no mesmo período, apontavam na direção da generalização do duplo caráter onda-corpúsculo à radiação, como também à matéria, evidenciando a indiscernibilidade dos estados idênticos e revelando, assim, propriedades fundamentais radicalmente novas que toda teoria quântica futura haveria de incluir.

O surgimento da mecânica quântica, nos anos de 1925-1927, consagrou o duplo caráter ondulatório e corpucular da luz e dos elementos da matéria no nível atômico, ao basear-se, por assim dizer, sobre esse caráter, ao menos em um primeiro momento. Essa dualidade constituiu um problema, durante muito tempo (e ainda hoje continua sendo 
problemática para alguns), uma vez que, segundo a física clássica, trata-se de dois caracteres incompatíveis no seio de uma mesma teoria física. Ela suscitou interpretações fisico-filosóficas sobre a natureza da descrição do mundo físico nesse domínio, a tal ponto que alguns chegaram a pôr em dúvida a própria noção de realidade física. Todavia o domínio do conhecimento que a teoria quântica atual adquiriu sobre os fenômenos e sobre os "sistemas" físicos de que eles são a fonte, no domínio quântico, levou a maior parte dos físicos a considerar as coisas de outra maneira, adotando, de fato, uma outra perspectiva em seus trabalhos e reflexões sobre a física, embora, às vezes, seja impossível fazer uma distinção entre os dois domínios, em virtude da impregnação dos antigos modos de pensar.

Segundo essa perspectiva, a onda e o corpúsculo, entendidos no sentido clássico usual dos termos, apenas são atribuíveis aos sistemas físicos (radiação, elementos de matéria) em uma abordagem em termos dos conceitos e das teorias físicas clássicas. No entanto, aprofundadamente, se pensarmos a partir da teoria quântica em sua forma atual, essas noções não são mais adequadas, e são substituídas na teoria quântica pelos conceitos ou grandezas propriamente quânticos, que são diferentes dos conceitos clássicos em suas formas e em suas relações mútuas. Esses conceitos ou grandezas efetivas têm a forma de "funções de estado" e de "operadores" (no sentido matemático), cuja utilização ou operacionalidade é codificada por regras bem definidas matematicamente, que lhes conferem uma significação física, tal como, por exemplo, o "princípio de superposição linear das funções de estados", que possibilita encontrar, ao fim de cálculos teóricos, a maior parte das propriedades especificamente quânticas (cf. Paty, 1999, 2000, 2003, 2008).

Afinal de contas, o caminho que possibilitou estabelecer tais noções, muito exatas e amplamente corroboradas pelos dados das experiências, mas, à primeira vista, pouco clássicas e intuitivas enquanto conceitos ou grandezas físicas apropriadas ao domínio quântico (aquele, em linhas gerais, que aparece desde o nível atômico muito afastado da percepção sensorial), não poderia deixar de ser difícil e indireto. Foi preciso que a "necessidade dos fenômenos", ' nesse nível de organização do mundo material, fosse progressivamente revelada pelo raciocínio e experiência, e encontrasse, no conhecimento físico, a formulação conceitual e teórica que lhes era a mais adequada. 
Isso foi feito por meio de uma série de etapas, entre as quais aquela que pôs em evidência, em 1923, o "efeito Compton" (cf. Compton, 1924), difusão elástica de raios X sobre elétrons atômicos, cujo papel foi considerado importante, já que contribuiu geralmente para convencer vários físicos de que a luz e toda radiação eletromagnética possuem as propriedades de um corpúsculo (energia e momento ou quantidade de movimento), embora manifestem também propriedades ondulatórias. Segundo as concepções admitidas até então, a luz era fundamentalmente de natureza ondulatória (a partir da teoria proposta por Fresnel que datava de mais de cem anos naquela época), assim como também era ondulatória toda radiação eletromagnética desde a teoria do eletromagnetismo de Maxwell.

A possibilidade de um caráter corpuscular da luz parecia em contradição com seu caráter ondulatório, verificado por todos os fenômenos conhecidos em óptica, que a teoria de Maxwell (modificada pela teoria da relatividade restrita, em 1905) ${ }^{2}$ explicava de maneira admirável. A ideia de uma quantificação da energia de radiação, e mais ainda, aquela de atribuir-lhe uma quantidade de movimento igualmente quantificada (isto é, discreta), que se opõe à ideia de continuidade das grandezas que subjazia a toda a física conhecida até então, era então vista mais como um retorno, dificilmente admissível, à teoria da emissão (de corpúsculos luminosos) de Newton, que a teoria ondulatória já havia descartado. Ora, na experiência realizada por Arthur Compton, a detecção do elétron ejetado do átomo e da radiação espalhada, e a determinação de seus parâmetros pela medida (que estava baseada, para os raios $X$, em suas propriedades ondulatórias e, para os elétrons, em suas energias e momentos), permitia fazer um balanço cinemático como em uma colisão de corpúsculos. Se chamamos " $\gamma$ " o raio $X$ incidente e " $e$ " o elétron (quase em repouso) no átomo, " $\gamma$ "” o raio $X$ que sai e " $e$ " o eletron ejetado, a reação se escreve $\gamma+e \rightarrow \gamma^{\prime}+e^{\prime}$. O equilíbrio de energias e de quantidades de movimento dava o resultado esperado para os corpúsculos, como na mecânica. Efetivamente, Compton interpretou sua experiência diretamente dessa maneira, indicando que um quantum de radiação electromagnética possui energia e momento $E=h v, p=h v / c(=h / c \lambda){ }^{3}$

2 Teoria que devemos a Einstein. Uma toria análoga, que pode ser denominada “eletrodinâmica relativista”, de Lorentz e de Poincaré, formulada paralelamente à de Einstein (em 1904-1905), modifica a teoria de Maxwell da mesma maneira que a de Einstein. As duas teorias eram consideradas, em geral, como equivalentes pelos científicos da época, até que a teoria de Einstein prevaleça, a partir do começo dos anos 1920, devido à sua perspectiva mais fundamental, manifestada por seu prolongamento, a teoria da relatividade geral, formulada no final de 1915 pelo próprio Einstein (cf., em particular, Paty, 1993,1996).

3 Sendo $h$ a constante de Planck (ver a nota 4 ), característica da quantificação, $v$ e $l$ respectivamente a frequência e o comprimento de onda ( $l$ inversamente proporcional à frequência $n$ ) da radiação considerada, os raios $X$, no caso da experiência de Compton. 
O efeito Compton foi geralmente considerado como a primeira prova experimental direta do momento quantificado e direcional da radiação eletromagnética, cuja necessidade Einstein havia mostrado em seu trabalho de 1916-1917, intitulado "Teoria quântica da radiação", ao qual ainda voltaremos. Quanto à energia quantificada, ela já havia sido formulada anteriormente, em 1905, por Einstein, radicalizando a hipótese feita por Planck, em 1900, de que as trocas de energia entre radiação e matéria são quantificadas (segundo a relação $D E=h n$ ). ${ }^{4} \mathrm{O}$ resultado obtido por Compton convenceu uma boa parte dos físicos que até então continuavam reticentes à ideia de quantificação da radiação eletromagnética. E principalmente o próprio Max Planck, que durante muito tempo tentou dissolver a descontinuidade das trocas de energia que ele havia revelado e, quando finalmente a admitiu (por volta de 1913), persistiu, até a realização da experiência de Compton, em ver nisso somente uma propriedade das trocas de energia nas interações entre a matéria e a radiação, e não a energia da radiação.

\section{III}

Evidentemente, é interessante saber como o próprio Einstein via esse resultado, que trazia o apoio da experiência a sua ideia sobre o "quantum de luz". Pelo que sabemos, há apenas um artigo escrito por ele a esse respeito, que não foi publicado em uma revista científica, mas em um jornal berlinense de ampla difusão, o Berliner Tageblatt, em 20 de abril de 1924 (cf. Einstein, 1924a). Esse artigo, que ficou posteriormente pouco conhecido, é muitas vezes omitido na literatura historiográfica. $\mathbf{5}$ Nisso reside, aliás, todo o interesse de sua republicação na língua original (o alemão) com uma tradução em espanhol (primeira tradução em língua latina), seguida de um comentário explicativo detalhado, ${ }^{6}$ que é um ensaio de estudo histórico, neste número de Scientiæ studia, colocando esse texto à disposição do leitor de hoje e oferecendo uma nova vida a esse momento histórico do pensamento científico, mais precisamente das ideias da física, da lenta e difícil elaboração das concepções quânticas, de que falávamos no início.

O artigo de Einstein é bem apresentado no comentário de "EyEC", e aqui pretendo apenas destacar sua formulação da problemática da experiência de Compton e de seu resultado. Após uma evocação histórica das teorias clássicas da radiação e dos

4. Sendo $D E$ a energia trocada entre a matéria e a radiação, $n$ um número inteiro, $n$ a frequência da radiação emitida ou absorvida, e $h$ uma constante universal, que, em seguida, será denominada "constante de Planck".

5 Espera-se, evidentemente, sua reprodução com os comentários de edição crítica em um volume a aparecer de suas obras completas (The complete works of Albert Einstein), publicadas na ordem cronológica por Princeton University Press. Esse texto foi omitido na tradução francesa (cf. Balibar, 1989).

6 Cassini, Levinas \& Pringe, 2013. Designaremos, daqui em diante, esse estudo por “EyEC”. 
conhecimentos adquiridos a partir da lei de Planck de quantificação da energia, devese constatar, explica Einstein, a necessidade de levar em conta as duas teorias físicas da radiação, "sem conexão lógica entre si" (Einstein, 2013 [1924], p. 217), a teoria ondulatória e a teoria dos quanta. A questão que aqui é formulada é, então, a de saber até que ponto deve-se atribuir aos corpúsculos de luz ou quanta as propriedades de projéteis, isto é, um momento dirigido. Ao fazer alusão, mas sem citá-lo, ao seu próprio trabalho de 1917, Einstein indica que essa questão teve já, há algum tempo, uma resposta afirmativa com base em considerações teóricas, e que a experiência de Compton provou a justeza (Richtigkeit) dessa predição (Voraussicht). Einstein conclui seu artigo, dizendo que "não apenas em relação à transferência de energia, mas também em relação aos efeitos da colisão, a radiação se comporta como se fosse constituída de projéteis discretos de energia" (Einstein, 2013 [1924], p. 219).

O estudo documentado "EyEC", que acompanha o artigo de Einstein, apresenta e discute o contexto científico das ideias da época sobre a física dos quanta de radiação na perspectiva da oposição entre o caráter corpuscular e o caráter ondulatório da radiação, em particular, as ideias de Compton e de Einstein, descrevendo seus respectivos trabalhos anteriores. Os autores do estudo propõem-se, em particular, a tentar analisar exatamente a formulação dada por Einstein, e entender por que ele escreve que a experiência prova que "la radiación se comporta como si consistiera en proyectiles discretos de energía", e não "la radiación es compuesta de proyectiles discretos de energía" (Einstein, 2013 [1924], p. 219), como foi admitido posteriormente, inclusive pelo próprio Einstein.

Aleitura desse trabalho, apesar de suas qualidades, gerou em mim certa insatisfação, o que motivou a redação desta nota crítica.

Em primeiro lugar, gostaria de indicar as razões dessa insatisfação a respeito de certos pontos do método seguido pelos autores do estudo. Em um segundo momento, quero retomar a exposição, reduzindo-a ao essencial, do estabelecimento dos conceitos quânticos durante o período que o estudo em questão abrange (ver-se-á então que as diferenças, em relação ao que foi proposto, não são apenas de nuances. Finalmente, desejo acrescentar elementos que não foram levados em conta no estudo supracitado sobre outros trabalhos e reflexões de Einstein, praticamente contemporâneos a seu artigo sobre o efeito Compton, que contribuem para o aprofundamento da compreensão de seu pensamento a respeito dos quanta, que se caracteriza por ser um pensamento em movimento. 
O estudo "EyEC" acantona-se unicamente na evocação dos quanta de luz, enquanto a introdução dos quanta na física concerne a um conjunto mais vasto de fenômenos, e o quadro de conjunto, que nos é fornecido sobre os quanta de luz, é, portanto, amputado de uma parte importante dos elementos de informação, tornando-se, assim, suscetível de deformar a apreciação dos conceitos em presença. Ora, somente levando em conta esse conjunto, mesmo que de maneira resumida, pode-se fazer uma ideia um pouco exata do papel e da importância dos "quanta de radiação" no estabelecimento da física quântica e das teorias que foram propostas a esse respeito. Isso concerne, particularmente, os trabalhos de Einstein. Por exemplo, a evocação do trabalho de 19161917, tal como foi feito no estudo "EyEC", concentra-se unicamente no resultado que concerne ao momento do quantum de luz, sem mostrar que esse resultado foi obtido por ter colocado em relação as propriedades já estabelecidas sobre as interações entre a matéria e a radiação e sobre a própria matéria. Veremos assim que, para Einstein, falar de quantum de radiação só tem sentido em relação a uma consideração mais ampla do conjunto dos fenômenos quânticos.

Em suma, essa escolha tem como efeito modificar o contexto histórico das ideias e distorcer muito a perspectiva, ao extrair, por assim dizer, o quantum de radiação do conjunto imbricado dos problemas da física quântica, tomando como intuitiva a ideia de corpúsculo luminoso, o que ela não era então de maneira alguma. (Isso se relaciona com a questão dos anacronismos, discutida mais adiante).

Por outro lado, o estudo em questão, ao querer limitar-se ao que se pode chamar de recepção por Einstein da experiência de Compton, opera um corte cronológico radical na data de publicação de seu artigo do Berliner Tageblatt, o que é, no mínimo, arbitrário. Pois, com toda evidência, a reflexão sobre os quanta não se interrompe em Einstein nesse momento particular, ela segue, por assim dizer, sua trajetória, e o conhecimento de seus outros trabalhos da mesma época ou ligeiramente posteriores, até 1925 - ano de sua última contribuição "construtiva" à teoria dos quanta, para retomar a expressão de Abraham Pais (1982) -, traz elementos complementares, ou até mesmo mais importantes, para a compreensão do que foi seu verdadeiro pensamento dos quanta, e dos quanta de luz, em particular. Nós o veremos, mais adiante, a propósito de suas reações à experiência de Bothe e Geiger, mais convincente do que a de Compton, e também com suas últimas contribuições, tão fundamentais, sobre as estatísticas quânticas e a indiscernibilidade das partículas idênticas.

Ao ficarem limitados ao quadro estreito que se fixaram, os autores não dispõem de todos os elementos necessários à reflexão, reduzindo, assim, o alcance de seu julgamento. Desse modo, várias vezes, eles espantam-se diante das formulações de Einstein 
a propósito da existência dos quanta de luz, que eles teriam preferido mais afirmativas, sobretudo, depois dos resultados publicados por Compton. Aliás, eles parecem criticar Einstein por não ter afirmado com mais clareza, mesmo antes de sua demonstração de 1916-1917, que o "quantum de luz" tinha todas as características de um corpúsculo, e opõem a sua demonstração do momento quantificado da radiação, fornecida nesse último texto, à formulação desse momento por Johannes Stark, a partir de 1909 (cf. Stachel, 1986).' No fundo, eles não estão longe de estimar que Stark havia precedido Einstein, quando da plena atribuição de uma quantidade de movimento da radiação. É surpreendente que não vejam a diferença entre, por um lado, adotar desde logo uma concepção puramente corpuscular (um modelo corpuscular) da luz, o que então Stark não hesitou em fazer, voltando à teoria da emissão de Newton e rejeitando, por isso mesmo, o que restava ainda, apesar de tudo, a levar em conta das propriedades ondulatórias (difração, interferências etc.) e, por outro lado, explorar de uma maneira racional e segura as propriedades dos fenômenos, sem fechar-se em uma hipótese que então só poderia ser arbitrária. O fato de que Einstein não quis impor, de inicio, uma natureza corpuscular à luz, enquanto ele o podia, já em 1905, $\mathbf{8}$ pode ser considerado como significativo de seu "estilo" de físico.

Outra limitação do estudo é o recurso, em várias passagens, a expressões anacrônicas e a sobreinterpretações, que, possivelmente, devem-se ao fato de que os autores tentam juntar dois pontos de vista que não conseguem conciliar: o da pedagogia e o da história. $\mathrm{O}$ aspecto histórico é evidentemente fundamental, e justifica a publicação do artigo "EyEC" na revista Scientiæ studia. Entretanto, ele é enfraquecido pelas escolhas arbitrárias, assinaladas anteriormente, e pelos anacronismos que agora apontamos. Talvez seja por razões pedagógicas que os autores propõem, várias vezes, formulações atuais para fenômenos ou conceitos que, na verdade, pareciam então obscuros, e cuja compreensão só foi possível posteriormente, uma vez contituída a teoria quântica.

Utilizada à propósito da quantificação unicamente da energia da radiação, proposta por Einstein em 1905, a expressão "quanta de luz" é literalmente inadequada; felizmente, os autores do artigo ponderam-na, destacando sua significação. Einstein fala, em seus primeiros trabalhos, de "quanta de energia da radiação", "quanta de energia de luz”, e não de “quanta de luz”. É verdade que rapidamente ele empregará essa

7 Todavia, eles reconhecem que Stark tem sua fonte de inspiração nas dicussões entre Einstein e Planck, quando do Congresso de Saltzburg daquele ano (cf. Einstein, 1909b).

8 Einstein mostrava, em sua teoria da relatividade restrita, que uma energia e uma frequência têm a mesma transformação de Lorentz, alguns meses depois de ter formulado a relação $E=h n$ para a radiação. Mas ele só destacou essa convergência ( $E / v$ é invariante de Lorentz, como a constante $h$ ) em seu trabalho de 19o9a, onde ele aborda, ao mesmo tempo, a relatividade e os quanta (ver mais abaixo). Uma análise dessa questão em termos de "estilo teórico", encontra-se em Paty, 1995 . 
última expressão, mas (salvo erro de minha parte) não antes de 1909, com a publicação do trabalho no qual ele evidencia, indiretamente, o índice de um duplo aspecto ondulatório e corpuscular da luz. Essa diferença é significativa, pois ela aponta, quanto às propriedades da luz, para além da granulação unicamente da energia (no entanto, o momento do raio luminoso somente seráfundado teoricamente nos trabalhos de 19161917). O "ponto de vista heurístico" adotado em 1905 prosseguia, por assim dizer, seu efeito de momento, em alguma medida, sobre a prospecção das propriedades físicas desse domínio da constituição da matéria, até então desconhecido.

Assinalamos rapidamente, a esse respeito, que Einstein não procede aqui (nem, em geral, alhures) por modelização, mas por exploração metódica. Ele não adotou, logo de início, um modelo corpuscular dos quanta de luz, e só foi progressivamente, por meio de desvelamentos sucessivos, que as propriedades corpusculares aparecem uma após outra; a segunda (o momento) esclarecendo a primeira (a energia), retirando-lhe um pouco de sua estranheza, ao referi-la a uma entidade melhor conhecida (a partícula dotada de energia e de momento), sem que, todavia, as dificuldades de compreensão, nesse estágio, sejam resolvidas, já que o corpúsculo deveria coexistir com a onda. Apenas uma nova teoria poderia pretender isso. Ele continuaria a fazer, nessa expectativa, o inventário das propriedades do domínio, que apontariam, mais adiante, a direção a seguir. Esse foi de maneira constante o método e o caminho de Einstein rumo à formulação de uma teoria dos quanta.

É evidente, em função do que acaba de ser dito, que o duplo caráter onda-corpúsculo para a luz, visto depois como uma dualidade, que nos é hoje familiar, não estava antecipadamente dado. Ele se confrontava com os fenômenos e também com as razões teóricas (Einstein descreve claramente essa situação em seu artigo sobre o efeito Compton). Para poder formulá-lo, era preciso criar uma nova acepção, que compreendesse principalmente as condições particulares apropriadas a sua utilização. $\mathrm{O}$ trabalho progressivo de Einstein caminhou diretamente nesse sentido, e é isso que o torna exemplar, como espero mostrar.

Einstein indicava claramente nos seus trabalhos que os quanta de luz são emitidos e absorvidos em suas interações com a matéria, e não somente difundidos na matéria, como o considera a teoria ondulatória. Os autores do artigo pensam que devem explicitar: "es decir, completamente creados o aniquilados, como Einstein ya había indicado en su explicación del efecto fotoeléctrico" (EyEG, p. 195). Mas não é isso que se lê nas contribuições de Einstein que tratam da física dos quanta. Se esses termos são usuais para nós hoje, eles não se encontram na física da época. Einstein, como seus contemporâneos, falava de "produção e transformação da luz" (em 1905), de "produção e absorção" (em 1906), de "absorção e de emissão" (em 1916-1917). Falar de "criação e aniquilação" seria já pronunciar-se, de alguma maneira, sobre a natureza do fenôme- 
no, até mesmo sobre sua explicação, enquanto ainda pairava sobre isso uma grande obscuridade teórica. Foi preciso esperar as primeiras formulações da teoria quântica dos campos, no início dos anos 3o, para que fossem levadas em consideração a criação e aniquilação das partículas quânticas de radiação e também de matéria, com o conhecimento das antipartículas e a utilização de operadores de criação e de aniquilação na formulação do campo quantificado. Essa terminologia é anacrônica para o período que precede. A preocupação pedagógica de explicar em termos atuais os conhecimentos do passado pode, se não se tomam certas precauções, apresentar graves inconvenientes do ponto de vista histórico, pois ela distorce as condições do aparecimento de ideias e de novos conhecimentos.

Os autores perguntam-se também sobre o fato de que Einstein, em vez de subscrever completamente o caráter de corpúsculo do quantum de luz, enfraquece sua conclusão, ao introduzir-lhe um condicional, "como se", e perguntam se não se pode ver aí o efeito de uma "concepción ficcionalista, o al menos anti-realista" (EyEC, p. 206), tirando esses conceitos de uma literatura recente, fornecendo, assim, uma "sobreinterpretação", sem que nada indique que ela seja necessária ou mesmo oportuna.

Mesmo admitindo que Einstein fosse antes predominantemente realista, eles acham que não é totalmente o caso para os quanta de luz, e remetem essa insatisfação a seus julgamentos sobre o caráter "incompleto" da teoria quântica (aliás, eles utilizam esse termo várias vezes, e até a propósito da quantificação da energia de 1905, no seu início). Isso também é um anacronismo, pois Einstein só falará de completude ou não da teoria quântica quando ela apresentar-se sob uma forma que possa ser considerada como uma teoria no sentido pleno do termo, isto é, sob a forma da mecânica quântica. E o termo adquiriu desde então, na física, um sentido preciso.

Antes disso, essa teoria estaria, a seu ver, ainda por construir, ou melhor, estaria em vias de construção, mesmo depois de ele mesmo ter proposto uma "teoria quântica da radiação" em 1916-1917 (cf. mais abaixo). Como se poderia então falar de completude a propósito de uma teoria que ainda não está bem formulada? Pode-se, certamente, notar uma continuidade nas exigências exprimidas por Einstein, entre a fase de elaboração teórica e a de crítica de uma teoria que se pretende satisfatória. E, notadamente, de uma para outra, a persistência de uma insatisfação quanto a não determinação de certas grandezas, do resultado da teoria de 1917, segundo a qual a direção da radiação emitida, munida de um momento quantificado é "deixada ao acaso", ao indeterminismo proclamado da mecânica quântica. Entretanto, colocar a exigência de completude antes do fim da construção, como ocorre no texto "EyEC", é colocar o carro antes dos bois. 
Uma vez feitas essas ressalvas, gostaria agora de delinear outra maneira de compreender a significação física do "quantum de luz", tal como ela poderia ser concebida, na época da descoberta do efeito Compton, mas também antes, seguindo o processo de investigação da física quântica e de elaboração das ideias teóricas que permitiu delimitar esse conceito e, em seguida, afirmá-lo. O caminho de Einstein, em suas pesquisas, é aqui evidentemente o melhor indicador, em vista da importância central de suas contribuições de então no domínio quântico, que constituem particularmente o interesse de sua reação ao anúncio de Compton.

$\mathrm{Na}$ época da descoberta do efeito Compton, a física quântica encontrava-se em possessão de uma boa parte dos conhecimentos que permitiriam que se cristalizasse em poucos anos (1925-1927) a teoria quântica propriamente dita sob suas duas formas, praticamente equivalentes, de mecânica ondulatória e de mecânica quântica. Entre as contribuições pioneiras das mais importantes e significativas pesquisas, as de Einstein figuravam no primeiro plano. Não é excessivo afirmar que, depois de seu trabalho de 1905 sobre a quantificação da energia luminosa, era ele quem dava o tom, muito antes até que seus colegas, inclusive Planck e Bohr.

Retomemos, de maneira breve, a série de contribuições de Einstein no campo da teoria dos quanta até a época de que tratamos (1923-1924). Elas mostram que o problema dos quanta - isto é, das propriedades da radiação, mas também das propriedades da matéria no nível atômico e das relações entre a matéria e a radiação -, foi, para ele, uma preocupação constante. Suas publicações sobre o assunto sucedem-se com regularidade de 1905 a 1925, isto é, até o momento da edificação da teoria quântica propriamente dita, mecânicas ondulatória e quântica, com uma diminuição relativa do ritmo de 1910 a 1915, que são os anos de seu intenso trabalho sobre a teoria da relatividade geral. ${ }^{9}$ Ele empreendeu, ao mesmo tempo, durante todos esses anos, suas pesquisas "heurísticas" 10 mais sistemáticas sobre a física dos quanta, e aquelas pesquisas que ele considerava, desde o início, mais "fundamentais" sobre as teorias do campo contínuo, que resultaram na formulação das teorias da relatividade restrita (em 1905) e geral (no fim de 1915), esta última propondo-se como teoria relativista geral da gravitação, com implicações cosmológicas

9 Baixa relativa, pois ele publicou, apesar de tudo, sobre o assunto, depois dos textos importantes de 1909, e antes daqueles de 1916, quatro artigos em 1910, um em 1911, três em 1912, dois em 1913, um em 1914, e nenhum em 1915, ano excepcional da última linha reta rumo à teoria da relatividade geral.

10 Segundo o próprio título de seu artigo, "Um ponto de vista heuristíco a respeito da produção e da transformação da luz" (Einstein, 1905). 
Einstein havia admitido, desde 1905, ao adotar um ponto de vista "heurístico"11 sobre a distribuição na energia da radiação, fornecida pela termodinâmica em analogia com a dos gases, que a energia associada a uma radiação eletromagnética de comprimento de onda dado $\lambda$ (e de frequência associada, inversamente proporcional, $v$ ) não é distribuída de maneira contínua, mas segundo valores discretos, $\varepsilon=h v$. Em 19o6, ele estabeleceu que os quanta de energia de radiação eram incompatíveis com a teoria ondulatória eletromagnética da luz, e obrigavam a considerar a necessidade de superála por uma outra teoria mais adequada, mas sobre a qual não se dispunha ainda de nenhuma indicação (cf. Einstein, 1905, 1906).

É a busca dos caracteres que deveria ter essa teoria, até então fora de alcance, que constituía, desde então, o eixo de seus trabalhos nessa direção, que exploravam as propriedades da radiação e da luz no domínio atômico. Deve-se dizer que ele havia concebido para isso, desde 1903, uma "ferramenta teórica" ou um método de investigação, fundado sobre a termodinâmica e a mecânica estatística de Boltzmann, capaz de interrogar as propriedades físicas de uma dada grandeza, levando em conta o fato de que as grandezas no nível atômico só nos podem ser fornecidas pela experiência segundo distribuições estatísticas. Ao tomar seu ponto de partida com a relação de Boltzmann para os gases entre a entropia e a probabilidade, $S=k \log W,{ }^{\mathbf{1 2}}$ ele considerava que a probabilidade $W$ deveria ter um sentido físico em vez de ser referida a um cálculo puramente aritmético de compleições, e definia a probabilidade como uma frequência de estado no tempo. A partir daí, em vez de acantonar-se no estabelecimento de valores médios, ele podia considerar que as flutuações em torno desses valores médios eram providas de um sentido físico, e que o cálculo de tais flutuações daria uma informação sobre certas propriedades das grandezas físicas consideradas. Foi assim que ele tratou o movimento browniano (em 1904-1905 com o sucesso que se sabe), e que ele foi levado, a respeito da radiação, a seus resultados de 1905 e de 1906, que acabamos de evocar.

A partir daí, Einstein empregou, de maneira sistemática, seu método, adaptando-o aos diversos fenômenos estudados, quase em todas as suas contribuições para a física dos quanta até 1925. Em 1907, e em seus trabalhos ulteriores, principalmente aquele apresentado ao Conselho Solvay do outono de 1911 (publicado em 1912), ele calculava os calores específicos dos átomos na hipótese de uma quantificação de sua energia e conseguia, assim, explicar seus valores em muito baixas temperaturas, consideradas até então como anormais, em relação à lei clássica de Dulong e Petit (cf. Einstein, 1907, 1912).

11 Ver o comentário que fizemos, mais acima, a respeito da utilização desse termo.

$12 S$ sendo a entropia, $k$ a constante de Boltzmann (assim denominada, depois de Planck), e $W$ a probabilidade de uma configuração (complexão) dada (cf. Einstein, 1903). 
Em 1909, pela primeira vez, ele examinou conjuntamente a teoria da relatividade restrita e as abordagens dos fenômenos quânticos. Einstein mostra, então, que há, na expressão teórica das interações radiação-matéria, o efeito de um duplo carater ondulatório e corpuscular (encontrando a adição de dois termos correspondentes na expressão de uma flutuação), e enuncia a possibilidade de que a radiação tenha uma quantidade de movimento orientada em uma direção, em vez de ser isótropa como o considera a teoria eletromagnética clássica. Ele também se interessou, embora momentaneamente, pela possibilidade de colocar juntas as duas propriedades, fazendo assim da radiação uma singularidade do campo (cf. Einstein, 1909a, 1909b). ${ }^{\mathbf{1 3}}$

No Conselho Solvay de 1911, Einstein proferiu uma conferência sobre os calores específicos, concluindo com a necessidade de uma teoria distinta da teoria clássica da radiação. Esse texto parece retrospectivamente ter sido muito importante para a aceitação final da quantificação da energia da luz, notavelmente através de Poincaré que, motivado pela comunicação de Einstein e pelas discussões, escreveu, pouco tempo depois, um artigo no qual demostrou o caráter inevitável da necessidade de encontrarse uma teoria não clássica; artigo esse que, por sua vez, teve muita repercussão no meio dos físicos (cf. Einstein, 1912; Paty, 2002).

Niels Bohr elaborou, em 1913, seu modelo do átomo nuclear com eletrons planetários cujas órbitas correspondem a níveis de energia quantificados, a emissão e a absorção de luz efetuando-se por saltos descontínuos de um elétron de um nível a um outro. Para tanto, baseou-se na quantificação das trocas de energia de Planck e também naquela dos estados de energia dos átomos, que havia sido estabelecida por Einstein em seus trabalhos sobre os calores específicos referidos acima.

\section{VI}

Einstein, por sua vez, retomou esta ideia de Bohr em 1916, calculando as amplitudes de transições entre os níveis de energia quantificados dos átomos no caso da absorção como da emissão direta da radiação. Ele se propôs, a partir daí, a encontrar a lei de Planck da radiação do corpo negro (forno aquecido a equilíbrio térmico entre a emissão e a absorção da radiação pelos átomos das paredes). Para obter esse resultado, ele precisava atribuir aos raios luminosos (electromagnéticos) não somente a energia quantificada que ele havia admitido em $1905(\varepsilon=h v)$, mas também uma quantidade de movimento (ou momento), direcional e quantificado $(p=h v / c(=h / c \lambda)$. A partir disso,

130 artigo "EyEC" indica, com toda razão, essas duas últimas considerações de Einstein, mas não como ele foi levado à primeira. 
concluía, pode-se "considerar como estabelecido de maneira relativamente segura" que a absorção e a emissão de radiação pelas moléculas materiais fazem-se não apenas com uma transferência de energia $(h v)$, mas também com uma transferência de momento $(h v / c)$, orientada entre a radiação e a molécula, sendo essa orientação incompatível com a teoria eletromagnética clássica (cf. Einstein, 1917, § ๆ). ${ }^{\mathbf{1 4}}$

Essa reunião coerente das propriedades características de fenômenos quânticos, conhecidos até então separadamente, evitando o quanto possível recorrer à teoria clássica da radiação, poderia justamente ser considerada como uma primeira abordagem de uma teoria dos quanta, pelo menos em um certo sentido, construtivo, do termo "teoria". Einstein intitulou esse trabalho "Teoria quântica da radiação", publicado, em sua primeira versão, em 1916 e, em uma segunda versão mais completa, em 1917. Essa teoria é conhecida desde então como "primeira teoria dos quanta", ou "teoria semiclássica dos quanta" (cf. Einstein, 1916b, 1917). ${ }^{\mathbf{1 5}}$

Todavia a teoria obtida continuava sendo ainda insatisfatória, a seu ver, porque não explicava o caráter corpuscular em relação à teoria ondulatória (caráter que seria contraditório em uma teoria clássica), e porque, por outro lado, ela fornecia apenas probabilidades de transição, deixando "ao acaso a duração e a direção dos processos elementares". Apesar dessas insuficiências teóricas (ou, pelo menos, diagnosticadas como tais por ele), ele destacava que "apesar de tudo, tenho plena confiança em que o procedimento aqui seguido seja seguro". O objetivo a ser almejado aparecia-lhe claramente dali em diante. "Essas propriedades dos processos elementares [implicadas pelo resultado obtido] tornam inevitável a formulação de uma verdadeira teoria da radiação", escrevia na conclusão do artigo de 1917. Mas ele também chamava a atenção para a exigência de que "toda futura teoria quântica da radiação deverá incorporar a transferência de momento da radiação", revelando, assim, exatamente sua própria estratégia na exploração dos fenômenos quânticos.

Tratava-se, portanto, para Einstein, não ainda da teoria propriamente quântica (cuja necessidade ele já havia exprimido de maneira cada vez mais insistente), mas mais modestamente de obter, como ele exprimiu mais tarde, "centelhas sobre os mecanismos de emissão e de absorção da radiação da matéria" (cf. Einstein, 1949a, p. 44). Com os quanta de luz providos de energia e de momento, o duplo caráter ondulatório e corpuscular da luz aparecia "como uma propriedade maior da luz e também, em geral, da matéria", escreveria Einstein sobre o assunto, retrospectivamente (cf. Einstein, 1949b).

14 A teoria fazia também aparecer um termo de "emissão estimulada".

$15 \mathrm{Na}$ época, a teoria de Bohr era, às vezes, designada como "teoria dos quanta". O próprio Einstein assim se referia a ela. Mas ela tem um alcance muito mais limitado do que a de Einstein, e responde antes ao qualificativo de "modelo (do átomo de Bohr)", nome que permaneceu. 
Em todo caso, é claro que, a seu ver, o problema fundamental colocado pelos quanta não estava resolvido. Por exemplo, ele escrevia a um correspondente, em 1921, ao evocar esses resultados: "evidentemente a questão dos quanta permanece em si mesma ainda igualmente obscura. Parece que nos defrontamos aqui com uma falha em nossas bases teóricas que nenhum pesquisador atualmente encontra-se em condições de demonstrar" (cf. Balibar, 1989, v. 1, p. 148-9).

É verdade que o artigo "EyEC" expõe o resultado nítido do trabalho de Einstein de 1916-1917, a atribuição de um momento quantificado e direcional à radiação. Mas se é indicado, com toda razão, que se trata de uma demonstração e não somente de uma hipótese, sua significação real, para Einstein, não é aqui facilmente apreensível. Ora, o momento está relacionado com a significação da teoria formulada, da qual ele é um elemento essencial. O que aqui está em jogo, parece-me, é o aspecto necessário dessa demonstração do momento da radiação, que se deve a sua natureza estrutural relativamente ao corpus das propriedades quânticas até então já bem estabelecidas. É, em particular, evidente que o pensamento einsteiniano do quantum luminoso é indissociável daquele sobre a constituição quântica da matéria. Um exemplo disso é o interesse de Einstein por outro fenômeno importante relativamente à aceitação dos quanta como significativos do ponto de vista da física, constatado pouco tempo antes da experiência de Compton, pela experiência de Stern e Gerlach sobre a quantificação dos momentos magnéticos dos átomos e sua explicação em termos quânticos (cf. Einstein \& Ehrenfest, 1922).

A subestimação desse aspecto deixa escapar o essencial do que era então a concepção teórica dos quanta, e, assim, não são oferecidos os meios para entender as razões do trabalho de Einstein.

É em virtude dessa posição central do momento quantificado da radiação na "primeira teoria dos quanta", que Einstein considerava ter o direito de declarar, a esse respeito, a seu amigo Michele Besso, em 1916, "a existência dos quanta de luz está estabelecida" (cf. Speziali, 1979).

Ao destacar a citação, os autores do artigo "EyEC" destacam, com razão, que se trata de uma declaração feita na esfera privada, e que não se encontra nenhuma outra semelhante nos artigos publicados (propriamente falando, a conclusão do artigo de 1916 afirmava isso de uma outra maneira, mais nuançada). Porém, não é muito difícil compreender a razão disso. Na esfera privada, Einstein evoca seus esforços, seu "combate" intelectual, para resolver o que ele chama também, de maneira privada, "o enigma dos quanta". As formulações científicas são evidentemente mais sóbreas, e atêmse aos elementos objetivos. 
Voltemos agora à experiência de Compton. A significação simples que esse autor lhe atribuía, em termos de colisão de corpúsculos, era, de fato, limitada no caso de elementos (átomos) leves, onde os elétrons atômicos são ligados de maneira fraca. Para os elementos pesados, era necessário levar em conta as forças de ligação dos elétrons atômicos, e a experiência de Compton nada disse sobre esse caso. Ela não era concluente também sobre o caráter individual ou apenas estatístico do balanço do momento-energia na colisão da radiação e do elétron. Consciente do fato de que a experiência apresentava limitações, o próprio Compton, assim como Bothe e Geiger, por outro lado, empenharam-se em superá-las.

O artigo "EyEC" não trata disso, em virtude dos limites que seus autores se impuseram. Eles concluem com uma dúvida quanto ao pensamento de Einstein sobre o assunto, enquanto poderiam ir além, se tomassem em consideração a continuidade de preocupações teóricas de Einstein sobre os quanta de luz (e de matéria). Eles se perguntam por que Einstein continua a dizer, mesmo depois da experiência de Compton, que os quanta comportam-se "como se" eles fossem corpúsculos, e consideram, finalmente, que lhe faltou audácia. No entanto (sem contar o que já apontamos anteriormente), se eles tivessem transposto um pouco sua barreira cronológica, eles teriam tido à mão os elementos para compreender melhor a posição de Einstein a respeito do efeito Compton. Parece já claro, em todo caso, que, para Einstein, a evidência do efeito Compton nada mais fazia do que confirmar seu resultado (teórico, convenho, mas significativo do ponto de vista físico), obtido em 1916-1917, ao qual ela não acrescentava grande coisa, salvo ilustrá-lo diretamente e trazer-lhe uma confirmação experimental relativa, mas ainda de maneira imperfeita. Ela não trazia elemento suplementar sobre essas propriedades e não contribuía, portanto, para fazer avançar a teoria necessária. Apesar disso, Einstein não deixava de "considerar os quanta luminosos como elementos de estrutura da radiação real", como escreve no outono de 1924 a um correspondente (carta ao senhor Magnus de 23 de novembro de 1924 apud Balibar et al., 1989, v. 1, p. 169).

As reações de Einstein, alguns meses mais tarde, ao anúncio da experiência de Bothe e de Geiger e àquela, um ano depois, de seu resultado, contrastam com sua moderação a respeito da experiência de Compton. É que a experiência de Bothe-Geiger possibilitaria ir mais longe, ao trazer um elemento de conhecimento suplementar, sobre o qual pairava uma incerteza, com respeito ao caráter individual das interações entre a radiação e a matéria e, portanto, da atribuição de um momento à radiação. Devido, precisamente, às insuficiências da experiência sobre o efeito Compton, Niels Bohr, Hendryk Anton Kramers e John Clarke Slater propuseram, em 1924, uma teoria (dita 
"teoria BKS") da difusão da radiação eletromagnética sobre os átomos com ejeção de elétrons, que se esforçava por preservar a teoria eletromagnética clássica e a continuidade nas trocas de energia no seio da estrutura do átomo, mas às custas do abandono da conservação estrita da energia e do momento, que teria apenas um caráter estatístico (cf. Bohr, Krammers \& Slater, 1924), banindo, assim, a noção de partícula material individual.

Walter Bothe e Hans Geiger propuseram-se, então, a realizar uma experiência em vista de testar a teoria BKS (cf. Bothe \& Geiger, 1924, 1925). Tratava-se de uma experiência, como a de Compton, sobre a difusão de raios $X$ sobre elétrons atômicos, mas dessa vez ligados em átomos complexos, e a determinação experimental dos parâmetros dos raios $X$ e dos elétrons será acompanhada, além disso, de uma medida da correlação entre a radiação espalhada e o elétron ejetado. Se, no resultado estatístico obtido, uma correlação forte aparecesse, ela significaria que a conservação da energia e do momento era verificada individualmente para cada interação, refutando, assim, a teoria BKS, e assegurando de maneira convincente que os raios $X$ (e, por extensão, a radiação eletromagnética) possuem individualmente uma quantidade de movimento, ou momento, quantificado.

Essa foi a razão pela qual Einstein foi levado a valorizar a experiência de Bothe e Geiger mais do que a de Compton, pois ela parecia-lhe a princípio mais concluente. Evocando essa experiência em andamento em uma carta a Paul Langevin, em dezembro de 1924, Einstein indicava que, para ele, o resultado seria uma correlação forte entre o elétron e a radiação e acrescentava que, se esse fosse o caso, dispor-se-ia então de "um novo argumento de valor em favor da realidade dos quanta de luz" ${ }^{16}$ Alguns meses depois, quando de sua viagem à América Latina (durante a qual ele passou um mês na Argentina, uma semana no Uruguai e uma outra no Brasil), Einstein escolheu esse assunto como tema de sua comunicação na Academia Brasileira de Ciências, recentemente fundada, e que o elegera membro correspondente. ${ }^{17}$ Nessa comunicação, intitulada "A situação atual da natureza da luz", que descreve o princípio da experiência de Bothe e Geiger, ainda aguardando seu resultado definitivo, Einstein afirma esperar desse resultado uma caracterização bem nítida da natureza corpuscular da luz que permitiria clarificar, sem equívoco, a questão do conflito entre as teorias ondulatória e corpuscular. Faltaria ainda conciliar esse caráter corpuscular da luz com as propriedades ondulatórias da radiação; em todo caso, ele era incompatível com a teo-

16 Einstein, carta à Paul Langevin do 16 de dezembro de 1924 (cf. Langevin, 1972). Veja-se também a carta ao Sr Magnus do mesmo ano, mencionada acima.

17 Einstein deu sua conferência em francês. O texto publicado então (Einstein, 1926) é uma tradução em português baseada no manuscrito original em alemão, que foi encontrado depois e publicado em 1997. Sobre as circunstâncias, e para uma análise detalhada dessa comunicação, que ficou pouco conhecida, cf. Paty, 2008. 
ria clássica, e a conciliação só poderia ser obtida na direção de uma teoria quântica que levaria em conta todas essas características.

O resultado da experiência de Bothe e Geiger, publicado em 1925, foi precisamente aquele que Einstein esperava; ele era claramente contrário à teoria BKS, e mostrou a forte correlação entre o elétron e a radiação nas interações; portanto, o caráter individual dessas últimas e, assim, do momento da radiação, isto é, o caráter plenamente corpuscular da radiação (É nessa época que foi proposto o termo "fóton", para dar conta desse dado).

Portanto, em definitivo, é a experiência de Bothe e Geiger, se alguma deve ser privilegiada, e seu resultado, referidos às restrições teóricas adquiridas, que devia confirmar a incompatibilidade dessa propriedade quântica com a teoria clássica e a necessidade inelutável de recorrer a uma outra teoria para substituir a primeira.

Esses acontecimentos precederam de pouco a verdadeira explosão teórica que constituíria o aparecimento da mecânica quântica, formulada em 1925-1927, e que se apresentou como a teoria procurada.

Destacamos a insistência de Einstein sobre o caráter individual dos processos de interação e das propriedades das entidades que neles intervêm. Parece claro que, para ele, não se pode falar de corpúsculo luminoso ou de radiação, a não ser se o corpúsculo e as grandezas que o caracterizam possuam um caráter individual, o que basta para explicar as nuanças ou as reservas que ele pôde emitir anteriormente ao emprego do termo "corpúsculo" na física dos quanta. Ele manteria sempre, a seguir, essa exigência, a saber, de aptidão da teoria para representar e descrever o fenômeno (e o "sistema") físico individual. Essa foi sua preocupação fundamental e o motor de suas críticas ulteriores da nova teoria quântica e de sua interpretação corrente ou dominante (cf. Paty, 1995, 2005, no prelo).

\section{VIII}

Além da experiência de Bothe e Geiger, outro desenvolvimento da física quântica, por volta da mesma época, traz um esclarecimento suplementar sobre o pensamento de Einstein a propósito dos quanta de matéria, mas também de luz ou de radiação. Já indicamos mais acima como o problema dos quanta de radiação não está isolado, e vinculase ao da quantificação dos estados da matéria. É significativo que, nas contribuições de Einstein sobre a física dos quanta, sua aceitação sem ressalvas do "quantum de luz como um corpúsculo", confortada pela experiência de Bothe e Geiger, acompanha, em paralelo, seus trabalhos sobre as propriedades quânticas dos gases monoatômicos, que serão suas últimas "contribuições construtivas" nesse domínio. Trabalhos fundamen- 
tais, já que se trata de nada mais nada menos do que do estabelecimento das propriedades específicas das estatísticas quânticas para a radiação e também para a matéria (pelo menos, para a matéria, no caso das partículas quânticas ditas de Bose-Einstein, de spin inteiro), que levava a considerar uma propriedade recíproca do caráter corpuscular da luz, o caráter ondulatório da matéria (cf. Einstein, 1924b, 1925a, 1925b; Bose, 1924a, 1924b). ${ }^{18}$ Sabe-se que foi Einstein quem indicou a Erwin Schrödinger a tese de Louis De Broglie sobre as ondas de matéria, da qual ele tomou conhecimento por intermédio de Langevin, e de cuja importância ele se deu imediatamente conta. No primeiro de seus artigos de 1925 sobre a estatística quântica, Einstein aproxima de maneira definitiva as duas propriedades.

Podemos pensar que, para ele, a estatística não clássica de partículas indiscerníveis, evidenciada desse modo, bem como a atribuição de comprimento de onda a um elemento da matéria, eram novas aquisições conceituais e que a teoria almejada deveria dar conta delas, mesmo sem que se compreendesse ainda suas razões profundas. Necessitava-se admiti-las como traços característicos fundamentais dos fenômenos quânticos, que se acrescentavam aos outros, ou que os traduziam. Apesar de não estar realmente satisfeito na sua compreensão dos fenômenos quanticos, pelo menos Einstein podia constatar que as propriedades desnorteantes dos quanta de luz não constituíam mais uma exceção; elas eram uma característica geral dos fenômenos quânticos. A ignorância das razões de ser desses últimos era, por assim dizer, diminuida de um grau, mais nem por isso estava superada. "Por mais que se corra atrás dos quanta, mais eles se escondem", escrevia ele em uma carta a Paul Ehrenfest em julho de 1924. (cf. Balibar, 1989, v. 1, p. 167). Ele poderia provavelmente ter dito o mesmo no final de 1925. Sómente a obtenção de uma teoria sintética e fundamental seria capaz de desvelálos verdadeiramente.

$\mathrm{O}$ “comportamento estatístico" não clássico dos elementos da matéria e da radiação, que Einstein referia à indiscernibilidade desses elementos, quando correspondem a estados idênticos (com a consequência, em particular, da predição da "condensação de Bose-Einstein”, que somente seria observada experimentalmente bem mais tarde, na última década do século xx), e sua conexão com as ondas de matéria, juntamente com a consideração da outra estatística quântica, a de Fermi e de Pauli (para as partículas de spin semi-inteiro), demonstrada quase no mesmo momento, deixavam o campo livre para a formulação da nova teoria quântica, que substituíria as teorias clássicas.

18 Sabe-se que Einstein assinalou à Erwin Schrödinger a tese de Louis De Broglie sobre as ondas de matéria (cf. De Broglie, 1925), que ele chegou a conhecer por intermediario de Langevin, dando-se imediatamente conta da sua importância. No seu artigo sobre a estatítica quântica (Einstein, 1925a), Einstein aproxima de maneira decisiva as duas propriedades (cf. Paty, no prelo). 
É interessante constatar que as duas primeiras formulações dessa teoria esperada, a mecânica ondulatória e a mecânica quântica, têm ambas, embora seguindo percursos diferentes, suas bases de partida na "teoria quântica da radiação", formulada por Einstein, em 1917. Abraham Pais (1982) indica isso precisamente, estabelecendo uma árvore genealógica das ideias e filiações a esse repeito. Mas isso seria outra história (cf. Paty, no prelo).

Em definitivo, os comentários e as contribuições de Einstein que seguem de perto seu artigo sobre o efeito Compton permitem compreender melhor sua posição exata sobre a questão da "existência dos quanta". A evidência empírica não lhe bastava, nem a formulação de modelos hipotéticos, era preciso dispor de uma teoria, que não mais seria a teoria eletromagnética clássica (ultrapassada, como ele havia demonstrado), capaz de dar conta das propriedades estabelecidas. Ele explorava os caminhos possíveis para recolher indícios sobre tal teoria e, primeiramente, sobre as propriedades características dos sistemas físicos que a teoria deveria incorporar.@

Traduzido do original em francês por Maria Aparecida Correa.

\author{
Michel Paty \\ Pesquisador do Laboratoire SpHere, UMR, \\ Centre National de la Recherche Scientifique (CNRS), \\ Universidade de Paris 7 - Denis Diderot - França. \\ michel.paty@univ-paris-diderot.fr
}

\title{
Remarks on Einstein's original approach towards a quantum theory of radiation (about the article "Einstein y el efecto Compton")
}

In the article "Einstein y el efecto Compton", published in this issue of Scientiæ studia, the authors wonder why Einstein did not claim unambiguously that this phenomenon was a clear and definitive proof of the corpuscular character of radiation. The aims of the present critical note are to provide additional comments that serve to clarify the method that Einstein used for exploring the quantum domain, in the absence of a satisfactory theory - a method he practiced from 1905 to 1925 , and from which he obtained evidence of new and unusual properties of quantum phenomena; and to try to formulate what he wanted to get at in his investigations, so as to understand better his 
thinking about quanta. Three main arguments are sketched. (1) The problem of light quanta cannot be separated from that of the quantum properties of atomic matter in general. (2) Another experiment, performed shortly after Compton's yielded a further element that was necessary to obtain a definitive answer to the question of whether a quantity of motion can be attributed to radiation. (3) Finally, Einstein's works on quantum statistics during that period pointed towards a generalization of the double wave-corpuscle behavior of radiation, and also of matter, providing evidence for the indistinguishability of identical states, thus revealing radically new fundamental properties that should be accounted for by any further quantum theory.

\section{REFERÊNGIAS BIBLIOGRÁFICAS}

AshteKar, A. et al. (Ed.). Revisiting the foundations of relativistic physics: festschrift in honor of John Stachel. Dordrecht: Kluwer Academic Publishers, 2003. p. 451-78. (Boston Studies in the Philosophy and History of Science).

Balibar, F. et al. (Ed.). Oeuvres choisies d'Albert Einstein. Paris: Seuil, Paris, 1989. $5^{\mathrm{v}}$.

Bohr, N; Kramers, H. A. \& Stater, J. G. The quantum theory of radiation. The Philosophical Magazine, 47, p. $785-822,1924$.

Bose, S. N. Planck's Gesetz und Lichtquantenhypothese. Zeitschrift für Physik, 26, p. 178-81, 1924a.

. Wärmegleichgewicht im Strahlungsfeld bei Anwesenheit von Materie. Zeitschrift für Physik, 27, p. 384,-90, 1924,b.

Bothe, W. \& Geiger, H. Ein Weg zu experimentellen Nachprüfung der Theorie von Bohr, Kramers und Slater. Zeitschrift für Physik, 26, p. 44, 1924.

. \&__. Über das Wesen des Comptoneffekts; eine experimentelles Beitrag zur Theorie des Strahlung. Naturwissenschaft, 13, p. 440-1, 1925 .

Cassini, A.; Levinas, L. \& Pringe, H. Einstein y el efecto Compton, Scientiae Studia, 11, 1, p. 185-209, 2013. (EyEG).

Compton, A. H. The scattering of $\mathrm{x}$-rays. Journal of the Franklin Institute, 198, p. 57-72, 1924.

De Broglie, L. Recherches sur la théorie des quanta. Annales de physique, 10a série, 3, p. 22-128, 1925.

De Broglie, M. \& Langevin, P. (Ed.). La théorie du rayonnement et les quanta. Communications et discussions de la réunion tenue à Bruxelles du 30 octobre au 3 novembre 19ı, sous les auspices de M.E. Solvay. Paris: Gauthier-Villars, 1912.

Einstein, A. Eine Theorie der Grundlagen der Thermodynamik. Annalen der Physik, 4, 11, p. 170-87, 1903.

. Ueber einen die Erzeugung und Verwandlung des Lichtes betreffenden heuristischen Gesischtspunkt. Annalen der Physik, 4, 17, p. 132-48, 1905.

.Zur Theorie der Lichterzeugung und Lichtabsorption. Annalen der Physik, 4, 20, p. 199-206, 1906. . Die Planck'sche Theorie der Strahlung und die Theorie der specifischen Wärme. Annalen der Physik, 4, 2, p. 180-90, 1907.

. Zum gegenwârtigen stand des Strahlungsprobleme. Physikalische Zeitschrift, 10, p. 185-93, 1909a. . Ueber die Entwicklung unserer Anschauungenüber das Wesen und die Konstitution der Strahlung. Physikalische Zeitschrift, 10, p. 817-25, 1909b. 


\section{Considerações sobre o Gaminho original de Einstein}

Einstein, A. Etat actuel du problème des chaleurs spécifiques, suivi de discussion du rapport. In: De Broglie, M. \& Langevin, P. (Ed.). La théorie du rayonnement et les quanta. Communications et discussions de la réunion tenue à Bruxelles du 30 octobre au 3 novembre 19ı, sous les auspices de M.E. Solvay. Paris: Gauthier-Villars, 1912. p. 407-50.

. Strahlung-emission und -absorption nach der Quantentheorie. Deutsche physikalische Gesellschaft Verhandlungen, 18, p. 18-323, 1916a.

Zur Quantentheorie der Strahlung. Physikalische Gesellschaft Mitteilungen (Zürich), 16, p. 47-62, 1916b.

. Zur Quantentheorie der Strahlung. Physikalische Zeitschrift, 18, p. 121-8, 1917.

Das Komptonsche Experiment, Berliner Tageblatt, 20 april 1924, Suppl. I. Trad. en espanhol : El experimento de Compton ¿Es la ciencia un fin en sí mismo?, neste numero de Scientiae Studia. 1924a . Quantentheorie des einatomigen idealen Gases, Preussische Akademie Wissenschaften, Phys. Math.

Klasse, Sitzungsberichte 22, p. 261-7, 1924,b.

. Quantentheorie des einatomigen idealen Gases. Zweite Abhandlung. Preussische Akademie

Wissenschaften, Phys. Math. Klasse, Sitzungsberichte, p. 3-14, 1925a.

. Quantentheorie des idealen Gases. Preussische Akademie Wissenschaften, Phys. Math. Klasse,

Sitzungsberichte, p. 18-25, 1925b.

. Observações sobre a situação actual da theoria da luz, Revista da Academia brasileira de sciencias, 1 , p. 1-3, 1926.

. Autobiographisches. Autobiographical notes. In: Schilpp, P. A. (Ed.). Albert Einstein, philosopher and scientist. La Salle: Open Court, 1949a. p. 1- 95.

. Reply to criticism. Remarks concerning the essays brought together in this cooperative volume. In:

Schilpp, P. A. (Ed.). Albert Einstein, philosopher and scientist. La Salle: Open Court, 1949b. p. 663-93.

. Das Komptonsche Experiment. Ist die Wissenschaft um ihrer selbst willen da? El experimento de

Compton ¿Es la ciencia un fin en sí mismo? Scientiae Studia, 11, 1, p. 211-9, 2013 [1924].

Einstein, A. \& Ehrenfest, P. Quantentheoretische Bemerkungen zum experiment von Stern und Gerlach, Zeitschrift für Physik, 11, p. 31-4, 1922.

Greffe, J. L.; Heinzmann, G. et Lonenz, K. (Ed.). Henri Poincaré. Science et philosophie. Science and philosophy. Wissenschaft und Philosophie. Congrès international, Nancy, France, 14-18 mai 1994. Berlin/ Paris: Akademie Verlag/Albert Blanchard, 1996.

Langevin, L. Paul Langevin et Albert Einstein d'après une correspondance et des documents inédits. La

Pensée, 161, p. 3-4.0, 1972.

Lindsay, R. (Ed.). Early concepts of energy in atomic physics. Dowden: Hutchinson/Ross, 1979.

PAIs, A. Subtle is the Lord. The science and the life of Albert Einstein. Oxford: Clarendon Press, 1982.

Paty, M. Einstein philosophe. La physique comme pratique philosophique. Paris: Presses Universitaires de

France, 1993.

The nature of Einstein's objections to the Copenhagen interpretation of quantum mechanics.

Foundations of Physics, 25, 1, p. 183-204, 1995.

Poincaré et le principe de relativité. In: Greffe, J. L.; Heinzmann, G. et Lorenz, K. (Ed.). Henri

Poincaré. Science et philosophie. Science and philosophy. Wissenschaft und Philosophie. Congrès international, Nancy, France, 14-18 mai 1994. Berlin/Paris: Akademie Verlag/Albert Blanchard, 1996. p. 101-43.

. Are quantum systems physical objects with physical properties? European Journal of Physics 20, p. $373-88,1999$.

. Interprétations et significations en physique quantique. Revue Internationale de Philosophie, 212, 2, p. 199-242, 2000. 
Paty, M. Poincaré, Langevin et Einstein. épistémologiques. Philosophie, sciences, histoire. Philosophy, science, history, 2, 1, 2, p. 33-73, 2002.

. The concept of quantum state: new views on old phenomena. In:AshteKar, A. et al. (Ed.). Revisiting the foundations of relativistic physics: festschrift in honor of John Stachel. Dordrecht: Kluwer Academic Publishers, 2003. p. 451-78. (Boston Studies in the Philosophy and History of Science).

. O Estilo científico de Einstein na exploração do domínio quântico; uma visão da relação entre a teoria e seu objeto. Scientiae Studia, 3, 4, p. 597-619, 2005.

Paty, M. Einstein e a física quântica no periodo da sua viagem à America Latina. In: Tolmasquim, A. T.; Almeida, M. \& Vergara, M. R. (Org.). Einstein para além do seu tempo. Bruchedo: Museu de Astronomia, 2008. p. 115-36.

. Einstein, les quanta et le réel. No prelo.

Schilpp, P. A. (Ed.). Albert Einstein, philosopher and scientist. La Salle: Open Court, 1949.

Speziali, P. (Org.). Correspondance Einstein avec Michele Besso (19o3-1955). París: Hermann, 1979.

StaGhel, J. Einstein and the quantum: fifty years of struggle. In: Colodny, R. (Ed.). From quarks to quasars. Philosophical problems of modern physics. Pittsburg: University of Pittsburg Press, 1986. p. 349-81.

Tolmasquim, A. T.; Almeida, M. \& Vergara, M. R. (Org.). Einstein para além do seu tempo. Bruchedo: Museu de Astronomia, 2008. 